\title{
Emerging Aspects of Robotics Technology
}

\author{
Nauman Khan \\ Department of Software Engineering \\ FUIEMS, Rawalpindi, Pakistan
}

\author{
Mateen Ahmed \\ Department of Software Engineering \\ FUIEMS, Rawalpindi, Pakistan
}

\begin{abstract}
There are high hopes that the planet will be full of robotics based computing machines. Robotics is based upon the philosophy of real time computing system that will work everywhere and new world come in place. Hard core areas which are under research in the domain of robotics is the handling with computational complexity and efficient responses to real time events for robot like machines. In this research paper computing application used in advance robotics have been presented and analyzed in a frame work of computational theory and domain of computing in general. Also the future trend that the advance robotics wills suffer is discussed.
\end{abstract}

\section{Keywords}

Robotics, Robot Manufacturing, Humanoid Robots, Classification of Robots

\section{INTRODUCTION}

Computing is usually defined to curriculum activity to improve and efficient the performance of the computer technology, like hardware and software. It is the most specific part of information technology. The designing and construction of software and hardware system is included in computing technology. A different kind of data builds and manages in a vast field of action. Computing will make computer system behave smartly developed communication and other entertainment media and collecting data related to some specific determination. Computing technology makes a machine mechanize for any particular activity that we can imagine. Automated computing rapidly changes how human are to find out problem solutions.

Computing when appeared was considered as the exceptional bounty for the elites of the society. At initial periods the computers lack the general public reorganization and were exclusively meant for cooperates or military use. With the emerging of new technologies a competition was observed in the computer development which accelerated the pace of computers application domain. With more and more application domain the computer even started the minute's task of human routine. With the presence of strong competition and the ever more acceptance of computers the concept o computer being for exclusive organization vanished and computers became a constituent of everyday life. In the present scenario the computers are doing even the very basic routine works. Now if you are not having a computer you are not worth of competing with the present technological era basic needs. Unfortunate reality is that while marketing the computer basic human comforts were neglected for the sake of financial gains. Such as designing of mouse was done neglecting the after effects of it on wrist of user.
This fig shows us the main computing component according to the other technology like robotics.

- Sensor

- Artificial intelligence

- Data storage

- HMI (human machine interface)

- Programming

This point is the domain of the embedded computing technology. In this era the embedded computing technology is frequently proceeding in the development country like US and JAPAN

In $20^{\text {th }}$ century computing changed the environment of the world. Computing was came into being like in the mid of $20^{\text {th }}$ century. Computers were introducing in the world due to the concept of computing. The first edge computers were operated by human operators. In the early of machine era the performance of the machine and its automation was not good, with the passage of time the computational technology was rapidly broad and it increases the performance, automation of the machine. In this era the result of self automated machine is robotics technology.

Robots are those physical machine which have programming approached that have some specific sensor and actuators etc. the manufacturer are given goal, for what purpose it should be used. In the computational approach algorithm process the input of sensor and a program control and manage the activities of the robot. How the robot should behave or work.

Demography trend in the US and other countries demand to increase and utilize of the robot. This trend was come in to being not only to solve the problem of fund social security as compared to the ratio or strength of older and much more retired people to younger worker increase. Robotics technology has good and worst effect on the environment of the world. In the era of $1970 \mathrm{~s} 1^{\text {st }}$ generation robots which consist of immobile, electronic and mechanical devices without sensors. In the mid of 1980 robots were build and it consist of sensors and s/w operator. In the mid of 1990 and the present Third-generation robots were developed. Which have advanced s/w and other important features related to the environment? The $4^{\text {th }}$ generation robots are under working and research phase, and computing approach features such as AI, Process of repeating fully automate, and nano \& micro size technology. Now a day robots are more advanced.Androids robots is one of the example of advance robots which has apparent similarity to human beings. Those types of robots are not the end of the robotics evolution.

Major types of modern robot:

- $\quad$ Fixed robots

- Mobile robot

In 2004, NASA landed twin exploration rovers on Mars named Spirit and Opportunity. This robot is the new sensing 
machine. This Robot was designed for the mission of carry out general geological examines of Martian rocks and earthly surface features. Both the rovers have exceeded their expected mission life by more than 20 times. On " 1 May, 2009"Spirit became stuck in soft soil which has prevented its movement but it continues to communicate with the earth station... Astronomical observations and atmospheric data have also been collected about the history of water on mars.

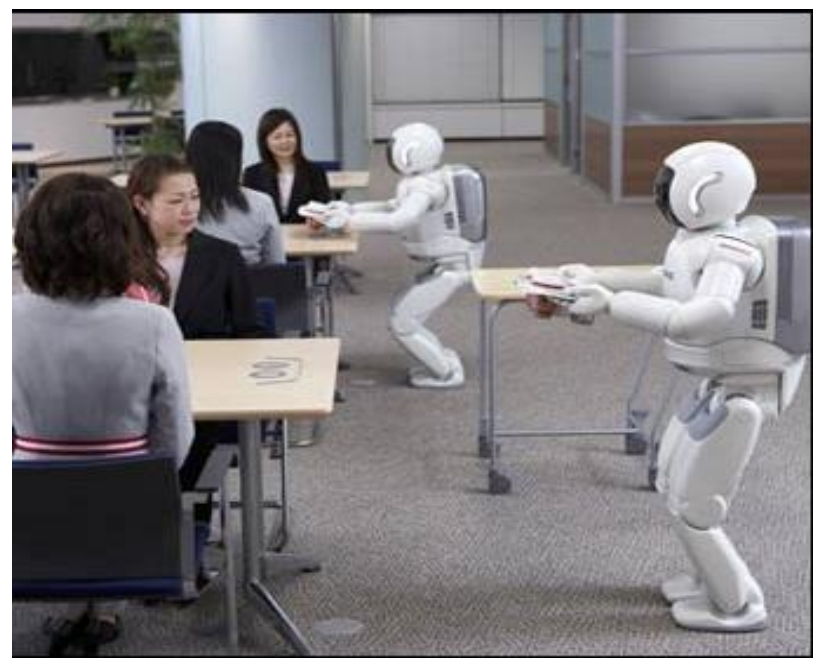

Figure - 1: Sony Humanoid Robots

The robot which is shown in fig 1, it is the first running robot. The running speed of that Robot is greater than $20 \mathrm{~cm} / \mathrm{s}$ and less than $24 \mathrm{~cm} / \mathrm{s}$, it is qualified for voice and face recognition. The battery life of that robot is approximately 55 min. QRIO robot is listed in world Guinness record book.

In this paper we describe the computing technology in robotics. The research roadmap of computing to describe a scope of critical capabilities in robotics manufacturing area is as under.

Fig-2 Shows the Research road map of computational techniques in Robotics .That techniques which achieved and impact on the critical capabilities and used in the Robotics technology [1].

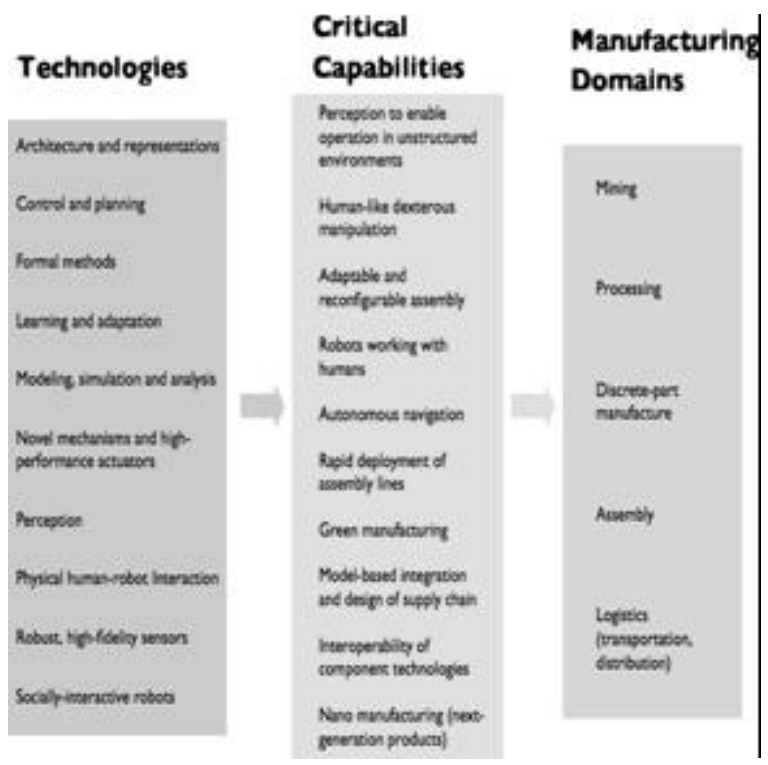

Figure - 2: Table of Mapping Techniques

\subsection{Architecture and Representation}

Modern robots must be intelligent and it memorizes something to their work experience. The operating system of robotics is supported such type of computing method like learning and algorithm models which is properly define behind their structure. High level reasoning is controlled to coupling of low level perception. A robot is not much liner and robots are automated to learn the skills with the jud gment to the human work. In this environmental situation the robots will need new method to adopt for performing the task automatically, without error and the recovery of error will be automate. [1]

\begin{tabular}{|c|c|}
\hline Deliberative & Reactive \\
\hline \multicolumn{2}{|c|}{ Speed of Response } \\
\hline \multicolumn{2}{|c|}{ Predictive Capabilities } \\
\hline \multicolumn{2}{|c|}{ Depentive } \\
\hline $\begin{array}{c}\text { World Model dependent } \\
\text { Slower response } \\
\text { High-level intelligence } \\
\text { Sophisticated planning }\end{array}$ \\
\hline
\end{tabular}

Figure- 3: Architecture overview in Robotics

In this fig the diagram show the architecture overview of robotics. The deliberative technique is a reactive approach to interacting with the world and Taking a purely, a reactive approach focuses on using the world as its own model and reacts, accordingly. To plans, reactive approaches depend upon finite state machines .to modify behavior as the world change. The advanced robots are much faster in their reaction times, and appearing quite intelligent in many scenarios.

\subsection{Control and Planning}

In Future Robots will more advanced as compared to current system. The greater automation in robotics will be possible due to their fast and efficient computational algorithm and extra features of control and planning. In future all robots will mobile. USA or other development countries will interested in the manufacturing of static base robot for the industrial application and similarity of most part of the algorithm of control and planning is founded in fixed robotics.

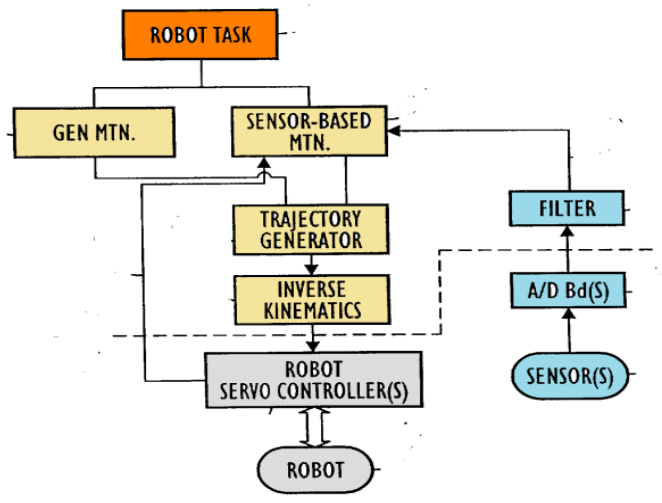

Figure - 4: Control flow chart of motion planner 
This fig shows the robot control $\mathrm{s} / \mathrm{w}, \mathrm{h} / \mathrm{w}$ and sensors. It provides the resulting plans provide by fine motion planner [4].

\subsection{Formal Methods}

In the domains of computing technology, different logic tools of computing is used for the guidance of robotics. These formal methods have been used in high level of application [1] .Such as humanoid robots application .However, the common use of formal methods in the robotics manufacturing phase. In present age Lack of usage of robotics technology worldwide this problem is also linked to the domain of important operations in robotics manufacturing.

\subsection{Learning and Adaptation}

In this technique, the sensors, software and other features is the main usage of robotics in factories is the work cells of engineering; this technique is used in Robots for performing their work in real life environments with greater AI . The learning procedure of robot like human by performing again and again the similar work is done with safely and matching their task actions and working results of the human's. Future Automated Robots can adaptable by control and checking their task actions and tuning to the limits of automation to manage their time working efficiently [5].

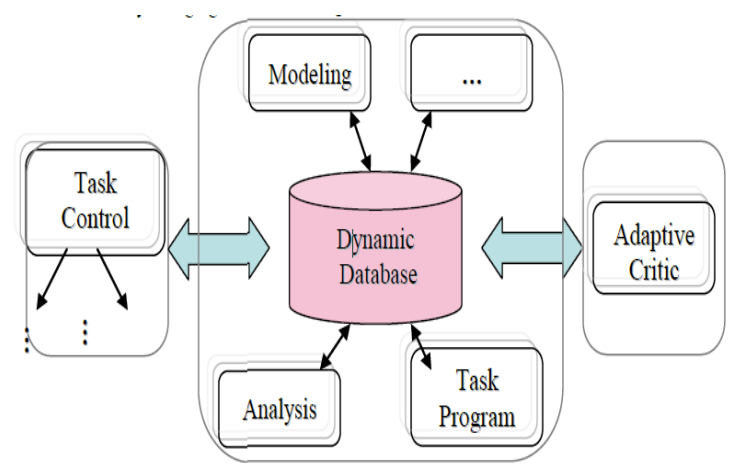

Figure- 5 Dynamic data base of Modern Robot

There are five important components in proposed diagram which has been following characterized:

- Task Control centre

- Dynamic Data base

- Critic network

- Model Based Action

- Action Network

The dynamic data base is show in fig 5 which resembles to a cylinder block in this fig, the dynamic data base creating the critic network for the training process and give surrounding area data to the system, these all activities is done in these algorithm. The structure of the environment is not normal for the operation of mobile robot and it is especially critical for the robotics technology. Another reason of the dynamic databases is following. GPS way points, for record the environment structured etc.

\subsection{Novel Mechanisms and High- Performance Actuators}

The performance of the modern robot is increase due to the novel mechanism and high performance actuators computational technique, so this is the Basic Topic which is needed in the fundamental research of computing in Robotics. Nano -and- micro scales part like MRI scanner due to that part environment shared with human which is applied in the domain of robotics application. In advanced Robotics, exoskeletons, smart prosthetics, and passive devices are the new procedure of human [6].

\subsection{Human-Robot Interaction:}

HRI (human robot interaction) is the big factor of the real time computing for the usage of Robotics technology. Therefore this technique will be more proceeding frequently and in the advanced robotics HRI factor are so better than previous robots, like humanoid robotics are more users friendly. The scientist worked on this factor to be more improved for the future robot interaction with humans and it's very simple to qualify according to their application capabilities. In present era of robots are adopted the new systems of sensing with advanced features, robots are able to that capture biometric data of human workers with advanced sensing mechanism .

Thus, Robots visual information depends on several factors: (i) the task a robot system is required to accomplish, (ii) visual sensors, (iii) required processing rate and (iv) Indoor/outdoor environment, to name some. In fig -8 it is a methods the use of vision in application domain of robot vision taking into account findings from biology, neuroscience, and cognitive science. In the end of this section we stress not only the individual functions in robot vision but also robot vision systems. In fig- 8 shows a tentative model of a robot vision system. The overview aims at indicating that, a robot vision system fulfills three major functions: navigation, grasping, and Human Robot Interaction (HRI). These task is depends upon the interplay function. For example, the computing technique of HRI and navigation is largely solved problem with methods suitable for applications and advanced topics open to research. [7]

\subsection{Perception}

This technique is used in the advanced robots due to the Artificial intelligence. An advanced robot is control the progress of their task and their perception system is much improved. In future we have great technique for heavy sensors and RGB camera which is the best method to perceive the image quality and information. According to computing in Robotics the necessary techniques include

- bio-metric sensor

- Human behaviour

- Human emotion

In the future Assembly strategies and movement of hand action is used as command in the computing world.

That how the advanced robots work according to their sensor and camera and navigation in perception is also the advanced research topic.

This fig shows the algorithm of robot detection by camera. It's the new way in perception of robotics and it's used in modern humanoid robot. In modern robots the $3 \mathrm{D}$ camera is placed in it and which read the face expression and it have other extra ordinary features related.

\subsection{Robust, High-Fidelity Sensors}

The sensory system is the revolution in microelectronics field, like Image sensors which provide good quality mega pixel images. Good signal quality is related to the domain of 
solid progress of sensor. Sensors are the most specific part in the robots which receive the input signals from the real environment. The new generation of sensor is made of silicon, which are the key aspects of future progress of robotics technology.

\section{Application Areas}

In now a day the robotics technology used in every aspect of life and it's made work easy for the human being in this planet. The advanced robotics is more efficient in performing the task as compared to human. This is all done by advanced and efficient computational technique. The computational technology progresses at every year.

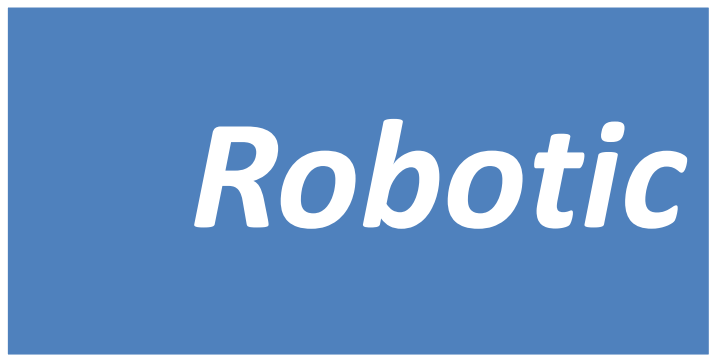

Figure- 6 Application of robotics

In fig 6 shows the basic application area of robotics .modern robotics perform their task in these area very well and the scientist work to increase their performance and more automated in these area. In this all application the computational technique is common which show in fig 1 is.

\subsection{MEDICAL ROBOTS}

In this era medical robotics is a part of the medical field. Today there are many operations are performed using new robotics technology, which reduce the effort of doctors in such types of operation. Robotics has many advantage in the medical field like minimally invasive surgery, including to reduce the patient spend time in the hospital, due to recovery of patient in less time. In short period of time, it improved the quality of care as much as possible, different typed of robots are used according to medical procedure. Robot is successfully interacting with human in the surgical, family room and rehabilitation centre [1].

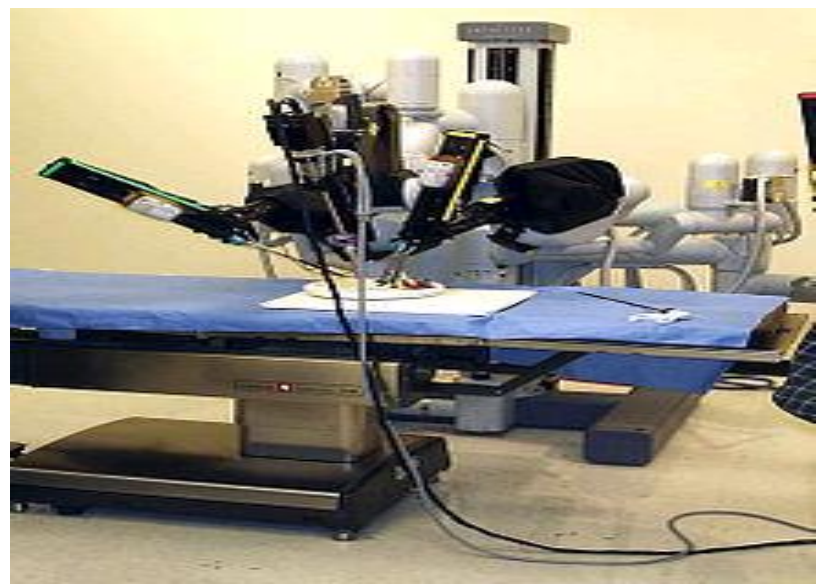

Figure- 7. Medical surgery robot

In this fig. you see the surgical robot machine which is automated work on command. it is the complex fixed robot machine which operate the patient quickly and efficient as human due to calculating algorithm. The engineer made the algorithm and program for these type robots very carefully because a little mistake is the bad luck for the patient.

\subsection{HEALTHCARE:}

In the hospital the patients who have feel serious pain and injury. The robotics is compulsion to have them hospitalized to ensure that they achieve full recovery within a given timeframe. More frequent sessions will be provided by robotic technology, in training there will be a higher level of adaptation, specific percentage of these training sessions will be soon available to perform at home. Most accurate and advance personalized treatment is maintained in this fashion by facilitating, the robotics technology will help in rehabilitation offers potential to complete patient recovery faster. In the beginning Robotics technology being used in health care for the early diagnosis of autism, and memory sickness etc, now a day's new products are available in the market, but still there is more to invent.

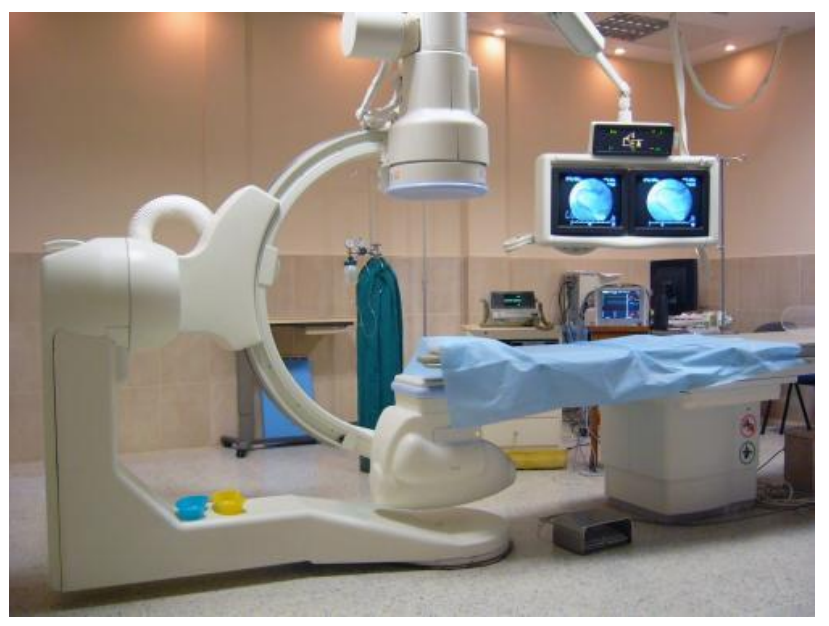

Figure - 8: Heal th Care machine

The fig -8 show the modern health care machine which come under the full grip of technology. This machine maintaining the health records of a patient, technically and more accurately. The following medical domains are influence due to this technology advancement:

\subsection{SERVICES:}

The robotics technology is used in the domain of service industry spans professional and domestic applications. Service robotics is improving by emerging applications of professional services. Cleaning, surveillance, and home assistance all of these are Domestic services applications. Service robotics had good effect on the economy of the country or big production companies. In the future of robotics technology the human work very less all of their work will done by service robots.

\section{LOGISTICS:}

Logistics processes are important part of our real life from each and every thing like transportation of product from factory to utility stores etc. Robotics technology has also the ability to better the checking process as well. In transportation and handling there are different phases for selling and delivery of items. The retail prices of food and other eatable things is related to transportation process. Modern robotics technology has the advanced computational 
technique ability to reduce the price of goods by logistic processes.

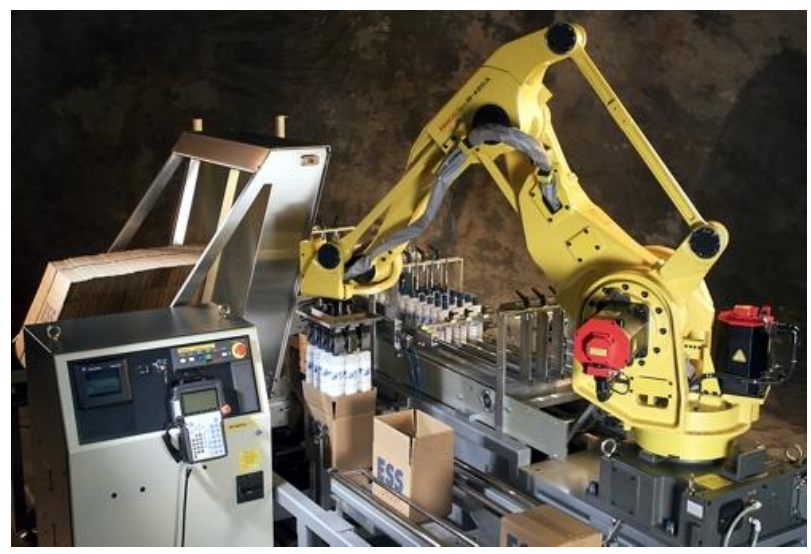

Figure- 9 Robotics arm for packing

The modern logistic Robot load unloads and packing the bottles, standardized packaged goods from contain er [1].

Today there is a large demand of logistic robots in market for business purpose. The working of the logistic robotics is to make the process of logistics more efficient, work easier. Business of the big companies is aware of the modern technology and they are interesting to investing for their business and it is benefited for them. The future of logistic robot will use every where.

\section{AGRICULTURE:}

The vision of advanced robotics technology in agriculture field is to increase the productivity rate of food items and reduce their prices '. Expert drivers are needed to drive complex agriculture machine efficiently because the usage of these machines is not easy, the productivity of these machines are usually lessen by labour cost and large expenses of machine operators. For all operation robots is being used such as crop spraying, harvesting, and all other operations which have been related to the other aspects points to agricultural to offered promise of small costs, increased protection, increased changeable operational system, and usage of small amount of chemicals. Pesticides use for fruit crop and crop harvesting; there are such prototypes and application which have been invented for the betterment of food quality and their production.

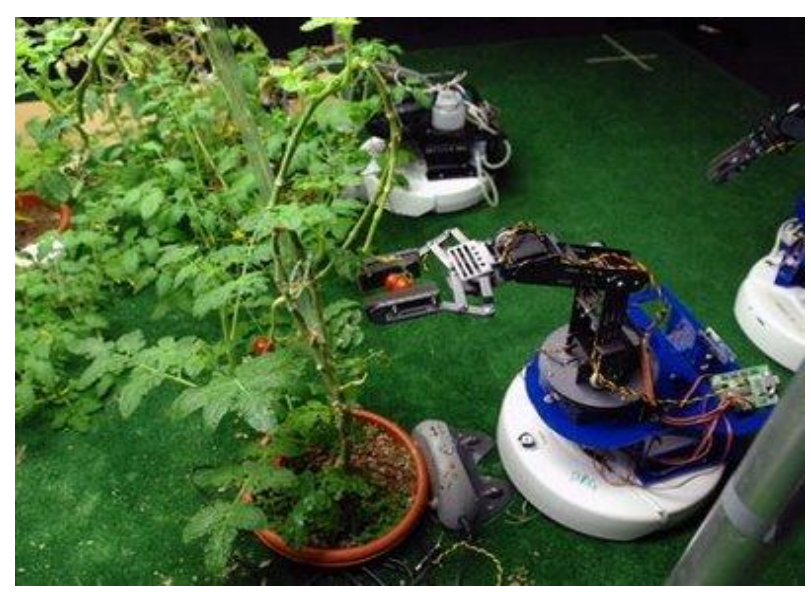

Figure- 10 Agriculture Robot
In fig 10 shows that robot is self automated which perform specific agriculture operation according to their computational logic and programming. In future the agriculture robotics is so efficient and due to their efficiency the productivity of items is more increase as compared to now a day [9].

\section{MINING}

In this era the underground and surface mining industries is fully depended on robotics technology. The mining robot which have a efficient working system and software layer algorithms is used in innovative belt inspection system ,that help the operators to detect errors and control the condition of part such conveyor belt and it is not fully automated, for example, it usage is increase for several underground coal mining. Foreign country recently announced the caterpillar's truck , which have potential to load 250 tons or more, into some mine sites.

\section{SECURITY}

In this computational era the computing technology like robotics is used for countries safety and protection which is continually increasing and growing as an innovative technology which has improved the, different functionality of different application like explosive counter measures and fire detection etc [1]. The use of robotic platforms by making of an unmanned surveillance, detection and response time like, fixed sensors and network are able to check the safety of boarders and locate the atomic threats. Personal robotic security systems will provide much better security to every individual. These types of robots contain with different technology. It has been used for different incidents such as flood and earthquake disasters.

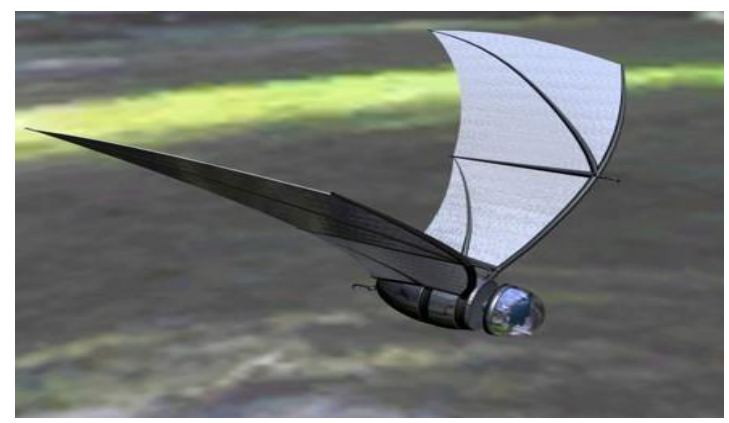

Figure- 11 War Fare robot

Scientist and Engineers envision making a micro spy plane robot, these types of micro robots collected information about enemy and inform to their soldiers. The researchers will seek attention and focus on the microelectronics and Nan-otechnology. The en gineers give the name of this robot to bat because it is look like bat. They invented in future which detect the sound in all direction and small sensor device will used due to the upcoming micro technology.

\section{TRANSPORTATION:}

In coming decades robotics technology will greatly affect the way how we transport different companies like Sag-way and Toyota have announced such a system which detect the rider's position and also help in their adjustment [1]. Powerful computer and sensors are installing in those smart cars which are creating by car makers and device manufacturer and give a good idea to the driver of their surrounding area and car efficiency with the help of sensors, cameras and automatic toll readers highway agencies are trying to develop smart road and a public-private national initiative smart cars. Now a day 
robotics technology adopt the Mass transportation systems to provide more awareness in the operators to handle critical situation more efficiently

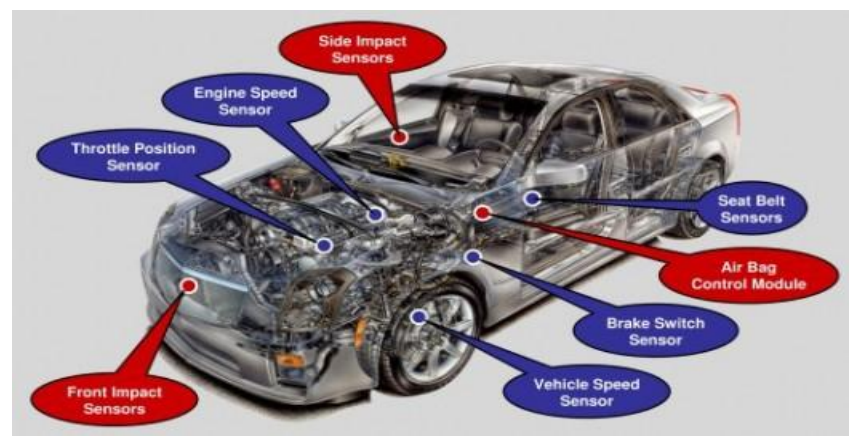

Figure - 12 Robotic Car

A large office building contains less microprocessor (tiny computers) than single modem automobile. Electrical and Computer Engineers installed these devices in robotic cars, which control every thing from the GPS navigation sy stem and fuel injection to anti-lock breaking to the environmental controls and sound systems are designed and fabricated.

\section{ENERGY \& ENVIRONMENT :}

According to researches energy and environment are two most exclusive issues for next generation in America and play important role in robotics technology applications. In the developed countries such as US and JAPAN, Robotics technology has helped a lot in inspection time without the involvement of advanced robotic technology is impossible to architect the separate sewerage system [1].

In Japan Shimizu corp. and yasu-kawa company is joint by the undertaking of the small robotics building project. in the future Using of discriminative system technology and robotics, the companies will interesting and making an computational real environment that can handle such duties as reception, deliveries, cleaning, and securities, without the need for human involvement that is very useful for countries future. Environmental robotics will be handling to the source network and it performs all functionality for the industrial and professional services.

\section{Potential Research Areas}

Cognitive computing is a dream for greater than 40 years. AI, clear logic, and neural networks are the success of conjunctive computing and a machine does not work and understand pictures as human.

According to Albus cites the following projects as proof that a future domain of the computing which is revolutionized is underway:

Modeling the brain: In 2008 researchers at IBM and Swiss government cooperation which vision to made a copy of a neocortical column,. This research will help repair damaged brains today.

Smart" cars: auto makers companies are interested to install heavily computerized safety features in their cars, researchers believe their future vision to save the life of people in driving due to their smart cars.

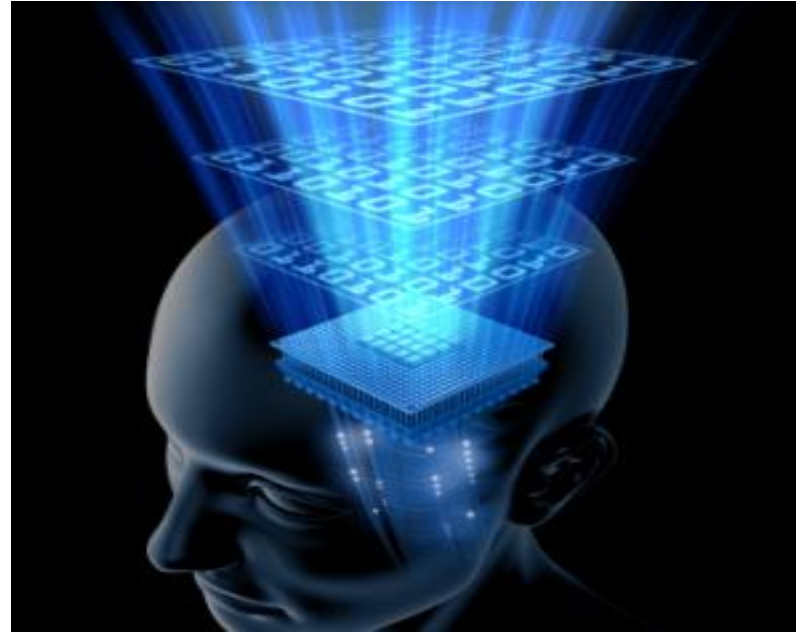

Figure-13 Human brain computing

Automating War: In the future, Department of Defense planners predict that all War field will be atomized and the risk of the lives of the solider in battlefield is much more saved example of this technology is auto-fly drones

Replacing aging neurons: In the early of 2030 cognitive computing working its way in our bodies, john Bruch give their perception, in the future we could be swap our brain cells with nanotech material to the absence of biological involvement. The level of thinking process will so fast at that level, $\mathrm{u}$ are not predicted [7].

In now a day in this planet the most research area on which the scientist and researcher work that is Nan-o-technology which increase and develop the computing technology and other technology like robotics and the 4th generation robots (Android), it is underdeveloped and this dream will be true it is not implemented at that time only knowledge about it. According to Nan-o-technology the future of the planet you can't imagine. It will be revolutionized the world and it effected on the life of the earth and human being as you can see the example of Nan-o-technology in the future.

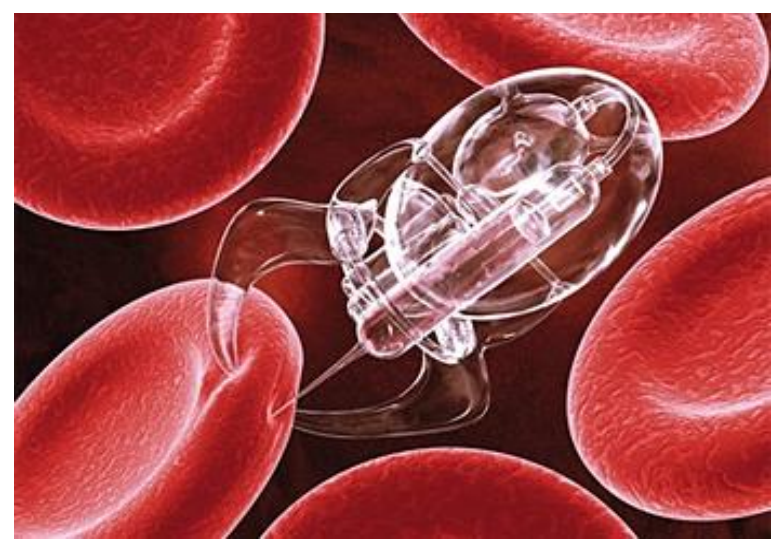

Figure-14 Nano- tech medical robot

Fig show the cancer diagnostic nano-robot which will be treated with ever blood cell of the human.

\section{CONCLUSION}

In this paper we discuss currently trend's techniques of computing technology in the robotics. This computing 
technique is used in the robotics and under developed in the domain of robotics technology, the scientist or researchers more work in that domain to make more efficient of robotics and computerized world, as we can imagine that planet may be at the top in the computing technology, We didn't know about the dream of the researcher that it will became true, we thinking the future technology is fantasy but it will come in to true and bring positive effect in your real life and the new computing technology is the way or gate to enter in the new world .

\section{REFRENCES}

[1] Computing Research Association and Community "A Roadmap for US Robotics from Internet to Robotics"

[2] Mi.sel Brezak a Ivan Petroviflc Eduard Ivanjko"Robust and accurate global vision system for real time tracking of multiple mobile robots" Department of Control and Computer Engineering, Faculty of Electrical Engineering and Computing, University of Zagreb, Croatia

[3] Robotics and Autonomous Systems 29 (1999) 205-226 "Integrated architecture for industrial Robot programming and control "Klaus Nilsson Rolf Johansson b

[4] US states Patent [19] Bourne et al. 11 patent no: 5,889,926 Date of patent: March 30, 1999

[5] "Engineering Robust Intelligent Robots" E. L. Hall, S. M. Alhaj Ali*, M. Ghaffari, X. Liao and M. Cao Center for Robotics Research
[6] "Artificial Intelligence - An Introduction to Robotics" Tim Niemueller and Sumedha Widya dharma July 8, 2003

[7] Jerry Pratt, Ben Krupp institute of Human And Machine Cognition, 40 South Alcaniz Street Pensacola, FL USA 32502 bY Robotics, Inc., 2138 Sinton Avenue, Cincinnati, OH USA 45206 "Design of a bipedal walking Robot

[8] International Journal of Social Robotics Manuscript No.(will be inserted by the editor) "Modeling Aspects of Theory of Mind with Markov" Random Fields Jesse Butterfield· Oddest Chadwick Jenkins · David M. Sobel Jona Schwertfeger

[9] "Multi-Feature Fusion in Advanced Robotic Applications "Zahid Riaz, Christophe Mayer, Michael Beetz, Bernd RadigSaquib Sarfraz

[10] "Robust and accurate global vision system for Real time tracking of multiple mobile robots" Mi.selBrezak o Ivan Petrovic EdouardIvanjko Department of Control and Computer Engineering, Faculty of Electrical Engineering and Computing, University of Zagreb, Croatia 